\title{
Technique For Estimating \\ Depths Of 100-Year Floods \\ In Pennsylvania
}

U.S. GEOLOGICAL SURVEY

Water-Resources Investigations Report 86-4195 
TECHNIQUE FOR ESTIMATING DEPTHS OF

100-YEAR FLOODS IN PENNSYLVANIA

By Herbert N. F1ippo, Jr.

U.S. GEOLOGICAL SURVEY

Water-Resources Investigations Report 86-4195

Harrisburg, Pennsylvania 
U.S. DEPARTMENT OF THE INTERIOR

MANUEL LUJAN, Jr., Secretary

U.S. GEOLOGICAL SURVEY

Dallas L. Peck, Director

For additional information write to:

District Chief

U.S. Geological Survey

4th Floor Federal Building

P.O. Box 1107

Harrisburg, Pennsylvania 17108-1107
Copies of this report can

be purchased from:

U.S. Geological Survey

Books and Open-File Reports Section Federal Center

Box 25425

Denver, Colorado 80225 


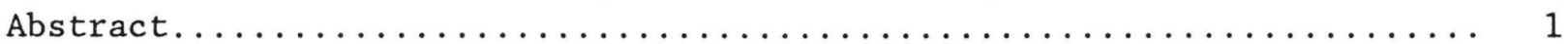

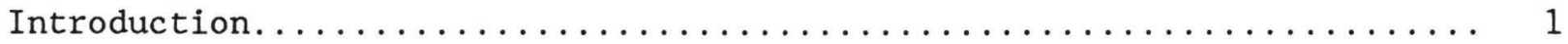

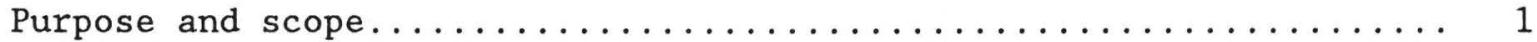

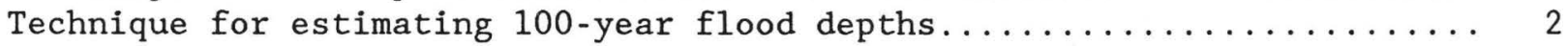

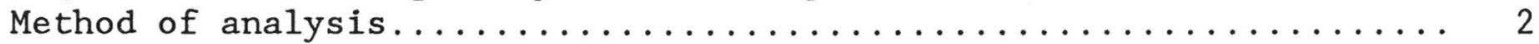

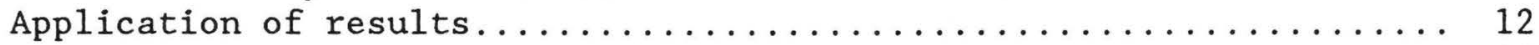

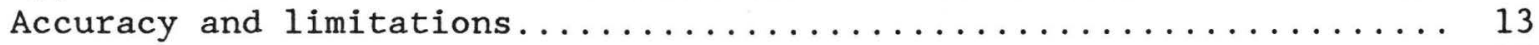

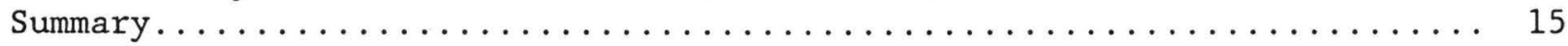

References cited.............................. 16

\section{ILLUSTRATIONS}

Plate [in pocket]

Plate 1.--Map of Pennsylvania showing locations of hydrologic areas and gaging stations

\section{Figures}

Figure 1.--Graph of relation between 100-year flood depth and drainage

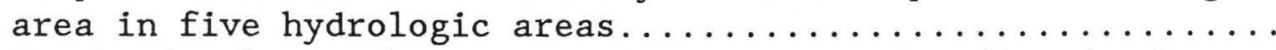
2.--Graph of relation between drainage area and median depth

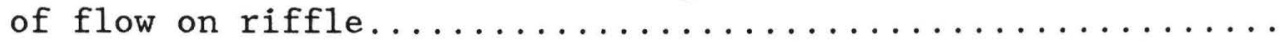

\section{TABLES}

Table 1.--Summary of equations for estimating 100-year flood depth.... 3 2.--Summary of gaging-station data.................. 5 
For the convenience of readers who prefer metric (International System) units rather than the inch-pound used in this report, the following conversion factors may be used:

Multiply inch-pound unit

cubic foot per second $\left(\mathrm{ft}^{3} / \mathrm{s}\right)$

cubic foot per second per square mile $\left(\mathrm{ft}^{3} / \mathrm{s}\right) / \mathrm{mi}^{2}$

foot ( $f t)$

foot per second ( $f t / s)$

inch (in.)

mile (mi)

square foot $\left(f t^{2}\right)$

square mile $\left(\mathrm{mi}^{2}\right)$
By

0.02832

0.01093

0.3048

0.3048

25.40

1.609

0.0929

2.590
To obtain metric unit

cubic meter per second $\left(\mathrm{m}^{3} / \mathrm{s}\right)$

cubic meter per second per square kilometer $\left(\mathrm{m}^{3} / \mathrm{s}\right) / \mathrm{km}^{2}$

meter (m)

meter per second $(\mathrm{m} / \mathrm{s})$

millimeter (mm)

kilometer $(\mathrm{km})$

square meter $\left(\mathrm{m}^{2}\right)$

square kilometer $\left(\mathrm{km}^{2}\right)$ 
By Herbert N. F1ippo, Jr.

\begin{abstract}
Techniques are developed for estimating 100-year flood depths in natural channels of unregulated Pennsylvania streams that drain less than 2,200 square miles. Equations and graphs are presented relating the depth of the 100-year flood above median stage to drainage area in five defined hydrologic areas in the State. Another graph defines the relation between drainage area and median depth of flow over the low point of riffles. Thus, 100-year depths on riffles can be estimated by summing depth values derived from two simple relations.
\end{abstract}

\title{
INTRODUCTION
}

Maps showing areas of potential flood hazards are useful tools in floodplain management. Preparations of such maps usually entails the estimation of flood depths, for a chosen recurrence interval, along stream channels or other paths of flow. Most commonly, flood profiles that correspond to 100-year flood depths along principal stream channels are developed to define, for mapping purposes, the spatial distribution of high-water elevations. The resultant map of 100-year flood boundaries delineates those areas having a probability of about 1 in 100 of being flooded in any year.

\section{Purpose and Scope}

The purpose of this report is to present a method, using simple relations, to estimate 100 -year depths of floods in natural channels of unregulated streams in Pennsylvania.

The depth of the 100-year flood above median stage--the stage corresponding to median (50th percentile) flow--can easily be estimated for unregulated streams throughout Pennsylvania. Mathematical relations, in which drainage area is the only independent variable, are provided for making such estimates for streams in five hydrologic areas. The range of application in three areas is from 1 to $2,200 \mathrm{mi}^{2}$ (square miles). The lower limit of applicability in the other two areas is also $1 \mathrm{mi}^{2}$; however, the upper limits are 325 and $560 \mathrm{mi}^{2}$, which approximates the areas of the largest drainage basins therein. A graph is provided for estimating median depth. 


\section{Method of Analysis}

The relation of flood depth to flood discharge, as developed by Leopold and Maddock (1953), is of the form:

$$
\mathrm{d}=\mathrm{cQ} \mathrm{Q}^{\mathrm{f}}
$$

where $d$ is the average cross-sectional depth, $Q$ is the discharge for a given probability of occurrence, and $c$ and $f$ are constants. Flippo (1977 and 1982) has shown that for probabilities of occurrence in the range of 0.5 to 0.01 for an annual event--corresponding to recurrence intervals of 2 to 100 years-there is a high correlation between unregulated flood discharges and drainage area for most streams in Pennsylvania. Consequently, for this study the equation of Leopold and Maddock was revised to a depth-predictive equation for unregulated streams of the form:

$$
\mathrm{D}_{100}=\mathrm{cA}^{\mathrm{f}}
$$

in which: A is the area of the drainage basin, $D_{100}$ is the depth of the 100year flood above the stage of median flow, and $c$ and $f$ are constants for the 100 -year flood.

Relations between flood depth and drainage area were developed in this study using graphical methods. Data on the depths, in feet, of 100-year floods above median stream stages were regressed against drainage areas ( $A$, in square miles) for 281 gaging stations on essentially unregulated streams. Regionalization procedures (Riggs, 1973) were used to minimize regression errors for five hydrologic areas (A-E), which are delineated on the map of plate 1. Gaging stations for which the stage-discharge relations are affected by channelization, or by backwater from bridges or other hydraulic controls, were omitted from this analysis. The drainage basins for several gaging stations lie in more than one hydrologic area. Each of these stations was grouped with those stations that served to define the hydrologic area in which the gage lies. Locations of the gaging stations are shown in plate 1.

Results of the regressions are summarized in table 1 and figure 1 . Data on drainage area, stages for median flow and the 100-year flood, and the depth of the 100-year flood above median stage are given in table 2. Owing to uncertainties in the definition of median-flow and 100-year-flood stages, computed depths of 100-year floods above median stages are less accurate than the hundredths-of-a-foot precision shown in this table.

Median stream stages (table 2) are those gage heights that correspond to median flows at the respective gaging stations. Median flows for continuousrecord stations were determined by magnitude-frequency analyses of daily mean discharges. Median flows at crest-stage partial-record stations were estimated from median-flow/drainage-area ratios for nearby continuous-record stations. Stage-discharge relations were used to translate the median flows into corresponding stream stages. 
Table 1.--Summary of equations for estimating 100-year flood depth [ft, feet; $\mathrm{mi}^{2}$, square miles]

\begin{tabular}{|c|c|c|c|c|c|c|c|c|}
\hline \multicolumn{2}{|l|}{$\begin{array}{l}\text { Hydrologic } \\
\text { area }\end{array}$} & Equation & ${ }^{1} \mathrm{~N}$ & $\begin{array}{l}\frac{\text { Areal }}{\mathrm{D}_{100}} \\
(\mathrm{ft})\end{array}$ & 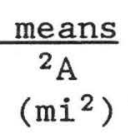 & \multicolumn{2}{|c|}{$\begin{array}{l}\text { Applicable } \\
\text { range in } \\
{ }^{2} \mathrm{~A} \quad\left(\mathrm{mi}^{2}\right)\end{array}$} & $\begin{array}{l}\text { Standard } \\
\text { error } \\
\text { (percent) }\end{array}$ \\
\hline A & ${ }^{3} D_{100}$ & $=1.80 \mathrm{~A}^{.380}$ & 15 & 7.19 & 38.2 & 1 & 325 & 24 \\
\hline B & $D_{100}$ & $=6.50 \mathrm{~A}^{.167}$ & 55 & 12.21 & 43.5 & 1 & $-2,200$ & 22 \\
\hline C & $D_{100}$ & $=3.72 \mathrm{~A}^{.241}$ & 155 & 9.80 & 55.7 & 1 & $-2,200$ & 25 \\
\hline D & $D_{100}$ & $=2.31 \mathrm{~A}^{.252}$ & 27 & 6.52 & 61.5 & 1 & $-2,200$ & 16 \\
\hline E & $D_{100}$ & $=2.87 \mathrm{~A}^{.261}$ & 29 & 7.52 & 40.0 & 1 & 560 & 28 \\
\hline
\end{tabular}

1 Number of gaging stations for which depth and drainage-area values were used in regression analysis. The arithmetic averages of these $\mathrm{N}$ values are the "areal means".

$2 \mathrm{~A}=$ Drainage area above site, in square miles.

$3 \mathrm{D}_{100}=$ Depth of 100-year flood above median stage, in feet.

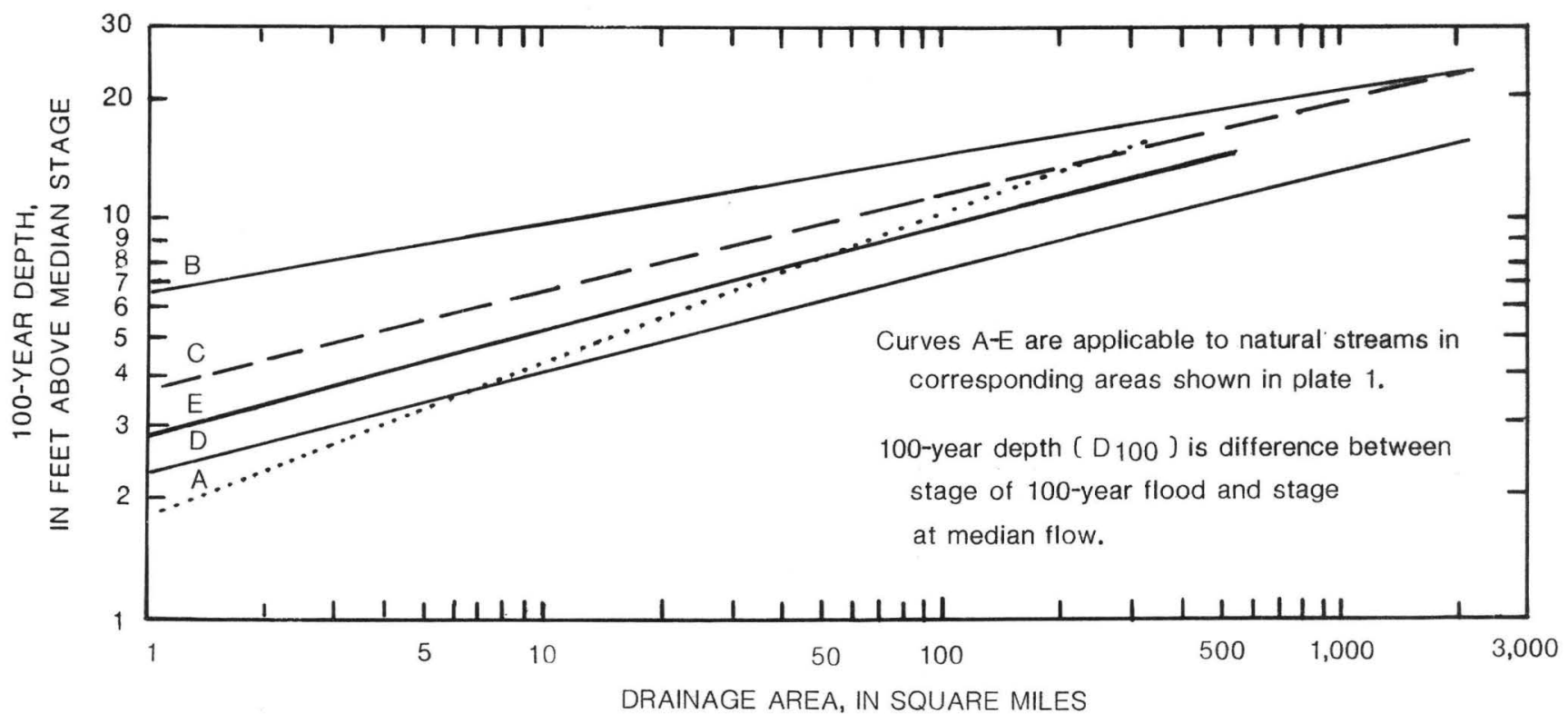

Figure 1.--Relation between 100-year flood depth and drainage area in five hydrologic areas. 
Stages for 100-year floods are those gage heights that correspond to 100 year instantaneous peak discharges. Station estimates of 100-year-flood discharges were computed through use of procedures set forth by the U.S. Water Resources Council, as revised by the Hydrology Subcommittee, Interagency Advisory Committee on Water Data (1982). Final estimates of 100-year-flood discharges were developed by weighting each station estimate with a regional estimate, as computed from a regional regression equation developed by Flippo (1982, table 3). The weighting procedure (Flippo, 1977, Appendix D) weights the two 100-year estimates in proportion to the number of annual peaks used to compute the station estimate and the equivalent years of record for the applicable regional regression equation.

Gaging stations for which there are fewer than 10 unregulated annual peak discharges of record, or for which the stage-discharge relation is not representative of natural hydraulic control in the channel, or for which the drainage area exceeds $2,200 \mathrm{mi}^{2}$, were not considered in this depth analysis.

A graph of drainage area versus median depth was prepared to facilitate the estimation of total 100-year depth. This graph (fig. 2) resulted from the least-squares fitting of median-depth values for 151 of the 281 gaging stations listed in table 2. Median-depth values were determined by subtracting the measured stage at the low point of the riffle that forms the low-water hydraulic control at the gaging station ("point of zero flow") from the stage at median flow (table 2). Only those measurements of riffle low points that were consistent with the stage-discharge relations used in the preparation of table 2 were considered. No reliable riffle measurements were available for many gage sites, including most partial-record stations. Riffle measurements for many other sites were unsuitable for this analysis, because the low-water controls are artificial structures. 
Table 2.--Summary of gaging-station data

[ ${ }^{\circ}$, degrees; ', minutes; $\mathrm{mi}^{2}$, square miles; ft, feet; $\mathrm{ft}^{3} / \mathrm{s}$, cubic feet per second]

Depth of 100

Station

number

Station name
Lati- Longi- Drainage Median flow

tude tude

$\left({ }^{\circ},{ }^{\circ}\right)\left({ }^{\circ},{ }^{\circ}\right)$ area

$\left(\mathrm{mi}^{2}\right)$ (ft) $\left(\mathrm{ft}^{3} / \mathrm{s}\right)$

100-year flood year flood

tage Discharge Stage Discharge above median

(ft) $\left(\mathrm{ft}^{3} / \mathrm{s}\right)$ stage (ft)

01431680 Mill Brook near Paupack

01432500 Shohola Creek near Shohola

01439500 Vandermark Creek at Mil

01440300 Mil.1 Creek at Mountainhome

01440400 Brodhead Creek near Analomink

01441000 McMichaels Creek at Stroudsburg

01442500 Brodhead Creek at Minisink Hill

01447500 Lehigh River at Stoddartsville

01447720 Tobyhanna Creek near Blakeslee

Lehigh River at Tannery

作

01450500 Aquashicola Creek at Palmerton

\section{North Branch Calkins Creek near Damascus}

01428500 Delaware River near Barryville, Hy

01429000 West Branch Lackawaxen River at Prompton

01429500 Dyberry Creek near Honesdale

01430500 Lackawaxen River at West Hawley

01430500 Middle Crek near Hatey

01431500 Lackawaxen River at Hawley

01451000 Lehigh River at Walnutport

01451800 Jordan Creek near Schnecksville

01453000 Lehigh River at Bethlehem

01458900 Tinicum Creek near Ottsville

01459500 Tohickon Creek near Pipersville

01465500

01465770

0146578

01465790

Neshaminy Creek near Langhorne

Poquessing Creek at Trevose Road, Philadelphia

Walton Run at Philadelphis

0146579

01467042

01467043

01467045

01467048

01467050

01467086 Creek at Grant Ave. Philadelphia Pennypack Creek at Pine Road, Philadelphia Stream 'A' at Philadelphia Pennypack Creek below Veree Road, Philadelphia Pennypack Creek at Rhawn Street, Philadelphia Wooden Bridge Run at Philadelphia

Frankford Creek at Torresdale Ave., Philadelphia

AREA

$\begin{array}{llll}41 & 23 & 75 & 14 \\ 41 & 27 & 74 & 55\end{array}$

$\begin{array}{llll}41 & 20 & 74 & 48 \\ 41 & 05 & 75 & 02\end{array}$

$\begin{array}{llll}41 & 05 & 75 & 02 \\ 41 & 10 & 75 & 16\end{array}$

$\begin{array}{llll}41 & 05 & 75 & 13 \\ 40 & 59 & 75 & 12\end{array}$

$\begin{array}{llll}40 & 59 & 75 & 12 \\ 40 & 60 & 75 & 09\end{array}$

$\begin{array}{lllll}40 & 54 & 75 & 12\end{array}$

$\begin{array}{llll}41 & 08 & 75 & 38 \\ 41 & 05 & 75 & 36\end{array}$

$\begin{array}{lllll}41 & 02 & 75 & 46\end{array}$

$\begin{array}{lllll}41 & 02 & 75 & 33\end{array}$

$\begin{array}{llll}40 & 54 & 75 & 30 \\ 40 & 48 & 75 & 36\end{array}$

AREA

4.84
83.6
5.36
17
5.84
65.9
65.3
59
10.4
91.7
18
22
2.39
49.9
76.7

$\begin{array}{lc}3.92 & 2.6 \\ 2.05 & 120 \\ 1.52 & 2.8 \\ 1.75 & 160 \\ 3.01 & 3.6 \\ 1.94 & 75 \\ 2.55 & 81 \\ 2.89 & 320 \\ 1.70 & 6.2 \\ .96 & 120 \\ 2.68 & 160 \\ 2.00 & 440 \\ 1.24 & 3.1 \\ 3.36 & 70 \\ 3.53 & 98\end{array}$

8.

$\begin{array}{ll}6.75 & 616 \\ 85 & 6.000\end{array}$

$\begin{array}{rr}4.20 & 668 \\ 10.05 & 10,800\end{array}$

$7.65 \quad 2,380$

$\begin{array}{rr}11.90 & 13,200\end{array}$

$17.85 \quad 45,100$

5.10
13.58
19,040

$15.80 \quad 14,800$

$19.20 \quad 41,200$

$4.37 \quad 851$

$\begin{array}{rr}14.20 & 6,460 \\ 12.80 & 9,920\end{array}$

$\begin{array}{lllll}41 & 42 & 75 & 10 & 7.02\end{array}$

$\begin{array}{lllll}41 & 31 & 74 & 59 & 2,020 \\ 41 & 35 & 75 & 20 & 59.7\end{array}$

$\begin{array}{lllll}41 & 35 & 75 & 20 & 59.7 \\ 41 & 38 & 75 & 16 & 64.6\end{array}$

$\begin{array}{rrrrr}41 & 38 & 75 & 16 & 64 . \\ 41 & 34 & 75 & 15 & 164\end{array}$

$\begin{array}{lllll}41 & 28 & 75 & 11 & 206\end{array}$

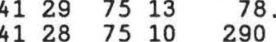

$\begin{array}{lllll}40 & 45 & 75 & 36 & 889\end{array}$

$\begin{array}{rrrrr}40 & 40 & 75 & 38 & 53.0\end{array}$

$\begin{array}{lllll}40 & 37 & 75 & 23 & 1,279 \\ 40 & 28 & 75 & 08 & 14.7\end{array}$

$\begin{array}{lllll}40 & 28 & 75 & 08 & 14.7 \\ 40 & 26 & 75 & 07 & 97.4\end{array}$

$\begin{array}{rrrrr}40 & 26 & 75 & 07 & 97.4 \\ 40 & 10 & 74 & 57 & 210\end{array}$

$\begin{array}{lllll}40 & 10 & 74 & 57 & 210 \\ 40 & 08 & 75 & 00 & 5.08\end{array}$

$\begin{array}{llll}40 & 05 & 75 & 00\end{array}$

$\begin{array}{llll}40 & 05 & 74 & 59\end{array}$

$\begin{array}{llll}40 & 04 & 75 & 00 \\ 40 & 03 & 74 & 59\end{array}$

$\begin{array}{llll}40 & 05 & 75 & 04\end{array}$

$\begin{array}{llll}40 & 05 & 75 & 04\end{array}$

$\begin{array}{llll}40 & 05 & 75 & 03\end{array}$

$\begin{array}{llll}40 & 03 & 75 & 02\end{array}$

$\begin{array}{llll}40 & 03 & 75 & 01 \\ 40 & 03 & 75 & 07\end{array}$

13.2

2.17
5.34

7.13

37.9

1.20
42.8

42.8
49.8

49.8
3.35

16.6

$\begin{array}{ll}2.25 & 3.7\end{array}$

$\begin{array}{rr}9.15 & 1,530 \\ 26.40 & 130,000\end{array}$

$\begin{array}{rr}26.40 & 130,000 \\ 12.40 & 10,200\end{array}$

$\begin{array}{rr}1.53 & 63 \\ 1.73 & 50\end{array}$

$\begin{array}{rr}1.73 & 50 \\ 1.10 & 160\end{array}$

1.78200

$\begin{array}{rr}.75 & 63 \\ 2.28 & 240\end{array}$

$2.81 \quad 1,400$

$2.151,600$

$\begin{array}{ll}1.07 & 5.7 \\ 1.32 & 37\end{array}$

$1.18 \quad 120$

1.50
1.71
2.41
1.89

1.89
2.40
2.92
3.10
9.75

9.75

3.98

2.39
3.22

3.22
2.27
3.71

3.
6.
1.
3.
3.
11
34
35
35
40
1.
14
2.

$16.70 \quad 16,100$

$20.60 \quad 28,000$

$18.10 \quad 31,600$

$21.97 \quad 42,500$

$19.70 \quad 90,390$

$30.20110,300$

9.80
10,230

$\begin{array}{ll}12.50 & 21,200 \\ 21.52 & 42,300\end{array}$

9.20
2,900

$11.40 \quad 1,850$

$13.30 \quad 2,960$

$13.70 \quad 4,760$

$12.48 \quad 7,590$

$\begin{array}{lll}.67 & 16.00 & 1,200\end{array}$

$\begin{array}{rr}15.20 & 8,960 \\ 13.00 & 11,400\end{array}$

$13.45 \quad 2,960$

$15.55 \quad 11,600$

$\begin{array}{r}2.83 \\ 6.20 \\ 2.68 \\ 8.30 \\ 4.64 \\ 9.96 \\ 8.75 \\ 14.96 \\ 3.40 \\ 12.62 \\ 13.12 \\ 17.20 \\ 3.13 \\ 10.84 \\ 9.27 \\ 6.90 \\ 23.23 \\ 10.87 \\ 14.97 \\ 19.50 \\ 18.32 \\ 17.35 \\ 19.69 \\ 16.89 \\ 11.27 \\ 28.05 \\ 8.73 \\ 11.18 \\ 20.34 \\ 7.70 \\ 10.99 \\ 8.99 \\ 11.41 \\ 11.30 \\ 12.33 \\ 9.38 \\ 6.25 \\ 11.22 \\ 10.61 \\ 10.23 \\ 11.23 \\ 11.84 \\ \hline\end{array}$

11.84 
Table 2.--Summary of gaging-station data--Continued

[ , degrees; ', minutes; $\mathrm{mi}^{2}$, square miles; $\mathrm{ft}$, feet; $\mathrm{ft}^{3} / \mathrm{s}$, cubic feet per second]

Depth of 100

Station

number

Station name

Lati- Longi- Drainage

tude tude

$\left({ }^{\circ},{ }^{\circ}\right)\left({ }^{\circ},{ }^{\circ}\right)$ area

$\left(\mathrm{mi}^{2}\right)$
Median flow

ge Discharge

(ft) $\quad\left(\mathrm{ft}^{3} / \mathrm{s}\right)$ 100-year flood year flood

charge above median

(ft) $\left(\mathrm{ft}^{3} / \mathrm{s}\right) \quad$ stage (ft)
01467500 Schuylkill River at Pottsville

Schuylkill River at

01470500 Schuylkill River at Bern

01471000 Tulpehocken Creek near Reading

01471510

01471980 Manatawny Creek near Pottstown

01472157 Fruph

01473000 Perkiomen Creek at Graterford

01473100 Zacharias Creek near Skippack

01473120 Skippack Creek near Collegevill

01473900 Wissahickon Creek at Fort Washington

01473950 Wissahickon Creek at Bells Mill Rd.

Philadelphia

01473980 Wissahickon Creek at Livezey Lane, Philadelphia

01474000 Wissahickon Creek at mouth, Philadelphia

01475300 Darby Creek at Waterloo Mills near Devon

01475530 Cobbs Creek at U.S. Highway 1, Philadelphi

01475550 Cobbs Creek at U.S.

01476000 Crum Creek at Darby

01476030 Little Crum Creek at Michigan Ave, Swarthmore

01476500 Ridley Creek at Moylan

01477000 Chester Creek near Chester

01478200 Middle Branch White Clay Creek near Landenberg

01480610 Sucker Run near Coatesvilie

01480617 West Branch Brandywine Creek at Modena

01481000 Brandywine Creek at Chadds Ford

01516500 Corey Creek near Mainsburg

01516800 Manns Creek near Mansfield

01518000 Elik Run near Mainsburg

01518500 Crooked Creek at Tioga

01520000 Cowanescue River near Lawrencevill

01531250 North Branch Sugar Creek tributary near Columbia Cross Roads

01532000 Towanda Creek near Monroeton

Douth Branch Towanda Creek at New Albany

01532850 Middle Branch Wyalusing Creek tributary near Richardville

01533250

01533500 North Branch Mehoopany Creek near Lovelton

01533800

Butter Creok at Gibson
AREA B--Continued

$\begin{array}{lllll}40 & 41 & 76 & 11 & 53 . \\ 40 & 38 & 76 & 08 & 133\end{array}$

$\begin{array}{lllll}40 & 48 & 75 & 58 & 42.9\end{array}$

$\begin{array}{lllll}40 & 31 & 75 & 60 & 355\end{array}$

$\begin{array}{lllll}40 & 22 & 75 & 59 & 211 \\ 40 & 20 & 75 & 56 & 880\end{array}$

$\begin{array}{lllll}40 & 20 & 75 & 56 & 880 \\ 40 & 16 & 75 & 41 & 85.5\end{array}$

$\begin{array}{lllllll}40 & 14 & 75 & 39 & 1,147\end{array}$

$\begin{array}{lllll}40 & 09 & 75 & 36 & 59\end{array}$

$\begin{array}{lllll}40 & 12 & 75 & 22 & 7.27\end{array}$

$\begin{array}{lllll}40 & 12 & 75 & 22 & 7.27 \\ 40 & 10 & 75 & 26 & 53.7\end{array}$

$\begin{array}{llll}40 & 07 & 75 & 13\end{array}$

40.8

$\begin{array}{llll}40 & 05 & 75 & 14\end{array}$

$$
\begin{aligned}
& 53.6 \\
& 59.2
\end{aligned}
$$

$\begin{array}{llll}40 & 01 \quad 7512\end{array}$

$\begin{array}{llll}40 & 01 & 75 & 25\end{array}$

$\begin{array}{llll}39 & 56 & 75 & 16\end{array}$

$\begin{array}{llll}39 & 59 & 75 & 17\end{array}$

$39 \begin{array}{llll}53 & 75 & 21\end{array}$

$3954 \quad 75 \quad 20$

$\begin{array}{llll}39 & 54 & 75 & 24\end{array}$

$\begin{array}{llll}39 & 52 & 75 & 25\end{array}$

$\begin{array}{llll}39 & 47 & 75 & 48 \\ 39 & 58 & 75 & 51\end{array}$

$\begin{array}{llll}39 & 55 & 2.57\end{array}$

$\begin{array}{llll}39 & 58 & 75 & 48 \\ 39 & 52 & 75 & 36\end{array}$

AREA C

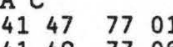

$\begin{array}{llll}41 & 49 & 77 & 06 \\ 41 & 49 & 76 & 58\end{array}$

$41497758 \quad 10.2$

$4154 \quad 7709 \quad 282$

$\begin{array}{lllll}41 & 59 & 77 & 09 & 298\end{array}$

$\begin{array}{lllll}41 & 50 & 76 & 50 & 8.83\end{array}$

$\begin{array}{lllll}41 & 42 & 76 & 28 & 215 \\ 41 & 35 & 76 & 26 & 13.3\end{array}$

$\begin{array}{lllll}41 & 52 & 76 & 00 & 5.67\end{array}$

$\begin{array}{lllll}41 & 42 & 76 & 07\end{array}$

$\begin{array}{lllll}41 & 32 & 76 & 09 & 35.2 \\ 41 & 48 & 75 & 39 & 7.38\end{array}$

$\begin{array}{cc}2.73 & 65.0 \\ 3.21 & 180 \\ 2.29 & 49 \\ 5.23 & 430 \\ 1.57 & 200 \\ 3.13 & 940 \\ 2.12 & 59 \\ 2.22 & 1,200 \\ 4.58 & 54 \\ 1.47 & 160 \\ 4.45 & 7.4 \\ 1.55 & 28 \\ 1.90 & 24\end{array}$

$14.50 \quad 9,470$

$10.99 \quad 7,520$

$18.82 \quad 42,000$

$17.10 \quad 19,600$

$28.60 \quad 71,000$

$13.6010,400$

28. 50 88,000

$20.30 \quad 48,600$

$10.80 \quad 10,200$

$18.30 \quad 21,400$

$\begin{array}{llll}3.34 & 37 & 13.70 & 9,720\end{array}$

$\begin{array}{rr}13.70 & 9,720 \\ 8.60 & 10,500\end{array}$

$\begin{array}{rrr}44 & 10.85 & 11,200 \\ 5.2 & 8.70 & 3,340 \\ 47 & 11.74 & 8,860\end{array}$

$\begin{array}{lll}47 & 11.74 & 8,860 \\ 3.7 & 12.20 & 5,490\end{array}$

$9.0 \quad 11.50 \quad 7,400$

$18 \quad 11.22 \quad 5,150$

$31^{.85} \quad 13.10 \quad 7,920$

$\begin{array}{llr}55 & 22.90 & 18,200\end{array}$

$\begin{array}{rrr}8.85 & 15.50 & 6,340 \\ 2.2 & 10.90 & 2,660 \\ 56 & 16.10 & 14,600\end{array}$

2.2

$\begin{array}{ll}16.10 & 14,600 \\ 17.00 & 26,100\end{array}$

$\begin{array}{lr}3.08 & 56 \\ 1.79 & 270\end{array}$

$\begin{array}{ll}1.68 & 3.4 \\ 2.92 & .85\end{array}$

$9.69 \quad 4,370$

$\begin{array}{rr}10.00 & 1,130 \\ 5.67 & 3,470\end{array}$

5.67
19.28
51,680

$18.50 \quad 48,200$

$\begin{array}{rr}1.61 & 120 \\ 2.61 & 31 \\ 7.22 & 78\end{array}$

$\begin{array}{ll}1.69 & 2.5 \\ 7.03 & 110\end{array}$

$\begin{array}{rr}6.82 & 3,320\end{array}$

$11.10 \quad 3,770$

$\begin{array}{ll}7.80 & 2,180\end{array}$

$8.62 \quad 3,130$

$9.95 \quad 14,500$
11.77

8.70

13.59

15.53

11.48

25.78

18.83

6.35

6.35
16.75
15.70

10.36

7.07

8.62
7.16

10.21

10.04

8.91

8.50
11.67

20.04

12.09

8.90

13.21

8.01

7.08
5.16
17.67

17.67

11.28

5.13
11.87

8.43

3.84
5.87

7.48
5.86 


\begin{tabular}{llcc} 
Lati- & Longi- & Drainage & \multicolumn{2}{c}{ Median flow } \\
& tude & area & Stage Discharge \\
$\left({ }^{\circ},{ }^{\prime}\right)$ & $\left({ }^{\circ},{ }^{\prime}\right)$ & $\left(\mathrm{mi}^{2}\right)$ & $(\mathrm{ft}) \quad\left(\mathrm{ft}^{3} / \mathrm{s}\right)$
\end{tabular}

100-year flood year flood

tage Discharge above median
AREA

01533950 South Branch Tunkhannock Creek near Montdale

01534000

01537500

01538000

01538800 near Tunkhannock Solomon Creek at Wilkes-Barre Wapwallopen Creek near Wapwallopen Huntington Creek near Pikes Creek

01539500 Fishing Creek near Bloomsburg Grove

01540000

01540200

01541000

01541500

ishing

ishing Creek at Bloomsburs

West Branch Susquehanna River at Bowe

Bradley Run near Ashville

01542000 Moshannon Creek at Osceol a $i 11 s$

0154272

0154350

01544450

01544500 Kettle Creek at Cross Fork

at Sterling Run ng Creek at Sinnemahoning Germania Branch at Germania 01547800 South Fork Beech Creek near Snow Shoe

01547950

01548020

01548500

Seech Creek at Monument

Pine Creek at Cedar Run

Pine Creek near Watervill

(1)

01549700 Pine Creek below Little Pine Creek near

Waterville

01550000 Laryon

01551000 Grafius Run at Williamsport

01552000 Loyalsock Creek at Loyalsockville

01552100 Mill Creek near Warrensville

Muncy Creek near Sonestown

01553600 East Branch Chillisquaque Creek near

01555000 Penns Cronville

01555500 East Mahantango Creek near Dalmatia

01555800 McDonald Run near East Freedom

Frankstown Branch Juniata River at Williamsburg

Little Juniata River at Tipton

Schell Run at Tyrone

01557500 Bald Eagle Creek at Tyrone

01558000 Little Juniata River at Spruce Creek

01559000 Juniata River at Huntingdon

Standing Stone Creek near Huntingdon

01560500 Dunning Creek at Yount
C--Continued $\begin{array}{llll}41 & 34 & 75 & 39 \\ 41 & 33 & 75 & 54 \\ 4 & 14 & 75 & 57\end{array}$ $\begin{array}{llll}41 & 14 & 75 & 57\end{array}$ $\begin{array}{llll}41 & 04 & 76 & 06\end{array}$ $\begin{array}{llll}41 & 19 & 76 & 09\end{array}$ $\begin{array}{llll}41 & 05 & 76 & 26 \\ 41 & 05 & 76 & 31\end{array}$ $\begin{array}{llll}41 & 05 & 76 & 31 \\ 41 & 00 & 76 & 28\end{array}$ $\begin{array}{llll}41 & 00 & 76 & 28 \\ 40 & 51 & 76 & 17\end{array}$ $4054 \quad 7841$ $\begin{array}{llll}40 & 31 & 78 & 35\end{array}$ $\begin{array}{llll}40 & 51 & 78 & 16\end{array}$ $\begin{array}{llll}41 & 13 & 78 & 35\end{array}$ $\begin{array}{lll}4125 & 2812\end{array}$ $\begin{array}{llll}41 & 19 & 78 & 05\end{array}$ $\begin{array}{llll}41 & 29 & 77 & 50\end{array}$ $\begin{array}{llll}41 & 23 & 77 & 41 \\ 4 & 0 & 77 & 54\end{array}$ $\begin{array}{llll}41 & 02 & 77 & 54\end{array}$ $\begin{array}{llll}41 & 07 & 77 & 42 \\ 41 & 00 & 77 & 20\end{array}$ $\begin{array}{llll}41 & 00 & 77 & 20\end{array}$ $\begin{array}{llll}41 & 31 & 77 & 27 \\ 41 & 19 & 77 & 23\end{array}$ $\begin{array}{llll}41 & 19 & 77 & 23 \\ 41 & 28 & 77 & 14\end{array}$

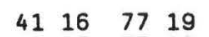
$\begin{array}{llll}41 & 25 \quad 77 & 19\end{array}$ $\begin{array}{llll}41 & 25 & 77 & 02 \\ 41 & 15 & 77 & 01\end{array}$ $4120 \quad 7654$ $\begin{array}{llll}41 & 20 & 76 & 58 \\ 41 & 21 & 76 & 32\end{array}$

$\begin{array}{llll}41 & 05 & 76 & 39\end{array}$ $\begin{array}{llll}40 & 52 & 77 & 03 \\ 40 & 37 & 76 & 55\end{array}$ $\begin{array}{llll}40 & 37 & 76 & 55 \\ 40 & 23 & 78 & 26\end{array}$ $\begin{array}{llll}40 & 28 & 78 & 12\end{array}$ $\begin{array}{llll}40 & 34 & 78 & 21\end{array}$ 40407815 $\begin{array}{llll}40 & 41 & 78 & 14\end{array}$ $\begin{array}{llll}40 & 37 & 78 & 08\end{array}$ $\begin{array}{llll}40 & 29 & 78 & 01\end{array}$ $\begin{array}{llll}40 & 31 & 77 & 58\end{array}$ $\begin{array}{llll}39 & 59 & 78 & 37\end{array}$ $\begin{array}{llll}40 & 04 & 78 & 30 \\ 40 & 03 & 78 & 28\end{array}$

12.6
383
15.7
43.8
4.94
274
56.5
355
1.77
315
6.77
371
68.8
8.34
272
685
2.40
136
46.2
12.2
152
1.99
604
750
37.7
944
6.80
173
3.14
443
11.9
23.8
9.48
301
162
1.54
291
5.58
93.7
1.68
44.1
220
816
128
5.28
172
191

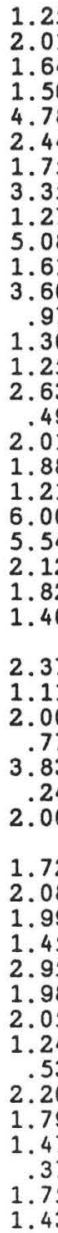

( $\mathrm{ft}^{3} / \mathrm{s}$ )

stage ( $f t$ ) 
Table 2.--Summary of gaging-station data--Continued

[ ${ }^{\circ}$, degrees; ', minutes; $\mathrm{mi}^{2}$, square miles; ft, feet; $\mathrm{ft}^{3} / \mathrm{s}$, cubic feet per second]

Depth of $100-$

\begin{tabular}{|c|c|c|c|c|c|c|}
\hline & & Lati- & Longi- & Drainage & Median flow & 100 -year flood \\
\hline $\begin{array}{l}\text { Station } \\
\text { number }\end{array}$ & Station name & $\begin{array}{l}\text { tude } \\
\left({ }^{\circ} \cdot{ }^{\circ}\right)\end{array}$ & $\begin{array}{l}\text { tude } \\
\left.\left({ }^{\circ},\right)^{\prime}\right)\end{array}$ & $\begin{array}{r}\text { area } \\
\left(\mathrm{mi}^{2}\right)\end{array}$ & $\begin{array}{l}\text { Stage Discharge } \\
\text { (ft) }\left(\mathrm{ft}^{3} / \mathrm{s}\right)\end{array}$ & $\begin{array}{l}\text { Stage Discharge above median } \\
\text { (ft) }\left(\mathrm{ft}^{3} / \mathrm{s}\right) \text { stage (ft) }\end{array}$ \\
\hline
\end{tabular}

01561000 Brush Creek at Gapsville

01562000 Raystown Branch Juniata River at Saxton

01562500
01563500
0 Juniata River at Mapleton Depot

01563800 Elders Branch near Hustontown

$\begin{array}{ll}01564500 & \text { Aughwick Creek near Three Spr } \\ 01565920 & \text { Lick Run near East Waterford }\end{array}$

01565920
01566000
Tuscarora Creek near Port Royal

01568000 Chler

01569000 Stony Creek nar

01569340 Stony Creek near Dauph

01572000 Lower Run at Newburg

01572900 Reeds Creek near Ono

01573000 Swatara Creek at Harper Tavern

01573500 Manada Creek at Manada Gap

$\infty$

01574500 West Conewago Creek near Manche

01575000

01576320

South Branch Codorus Creek near York

Codorus Creek near York

01576500 Conestoga River at Lancaster

01577 Muddy Creek at Castle Fin

01577940 Broad Creek tributary at Whiteford, MD

01578200 Conowingo Creek near Buck

01600700 Little Wills Creek at Bard

01601000 Wills Creek below Hyndman

01613050 Tonoloway Creek near Needmore

01619000 Antietam Creek near Waynesboro

03007800 Allegheny River at Port Allegany

03008000

03009680

Newell Creek near Port Allegany

03010500 Allegheny River at Eldred

03010655 Oswayo Creek at Shinglehouse

03028000 West Branch Clarion River at Wilcox

03029000 Clarion River at Ridgway

03029200 Clear Creek near Sigel

03029500 Clarion River at Cooksburg

03031950 Big Run near Sprankle Mills

03032500 Redbank Creek at St. Charles

03034000 Mahoning Creek at Punxsutawney

03035000 Mahoning Creek near Dayton

03038000 Crooked Creek at Idaho

03039000 Crooked Creek at Crooked Creek Dam

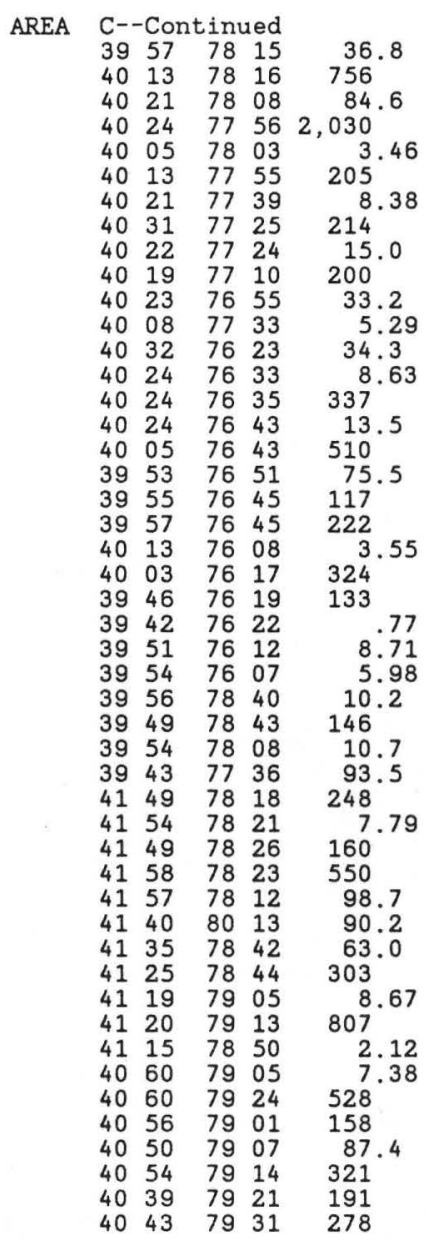

$\begin{array}{lc}2.25 & 21.0 \\ 1.88 & 400 \\ 1.59 & 42 \\ 3.39 & 1,200 \\ 3.96 & 1.05 \\ 3.26 & 90 \\ 2.70 & 4.2 \\ 3.62 & 110 \\ 2.91 & 7.5 \\ 1.48 & 130 \\ 1.57 & 38 \\ 1.65 & 3.7 \\ 1.31 & 30 \\ 1.42 & 5.4 \\ 1.99 & 310 \\ 1.70 & 14 \\ 3.17 & 230 \\ 2.20 & 43 \\ 1.18 & 68 \\ 2.32 & 120 \\ .60 & 2.6 \\ 3.50 & 240 \\ 3.57 & 120 \\ .01 & .58 \\ 2.41 & 6.6 \\ 2.51 & 4.5 \\ 5.80 & 3.1 \\ 2.30 & 60 \\ 3.06 & 4.5 \\ 3.30 & 88 \\ 2.97 & 275 \\ 2.55 & 5.5 \\ 2.92 & 190 \\ 3.80 & 450 \\ 4.21 & 96 \\ 2.45 & 45 \\ 1.83 & 64 \\ 2.93 & 310 \\ 2.91 & 5.4 \\ 3.10 & 800 \\ 1.90 & 1.6 \\ 2.04 & 5.4 \\ 3.75 & 400 \\ 1.82 & 135 \\ 2.00 & 68 \\ 2.65 & 260 \\ 2.83 & 120 \\ 1.45 & 160 \\ & \end{array}$

11.00
21.71
8.45
34.06
9.85
19.80
10.40
21.15
10.40
20.40
11.87
6.91
10.50
9.90
21.50
11.00
21.16
14.00
20.30
23.00
9.20
20.78
22.80
3.00
11.50
9.90
11.00
13.10
11.60
15.10
23.00
6.60
17.15
26.50
19.00
15.55
12.65
22.20
8.95
18.60
8.20
6.94
19.00
18.30
14.60
14.00
17.30
19.50

\begin{tabular}{rr}
7,330 & 8.75 \\
59,000 & 19.83 \\
8,100 & 6.86 \\
126,600 & 30.67 \\
829 & 5.89 \\
26,900 & 16.54 \\
2,720 & 7.70 \\
22,300 & 17.53 \\
8,820 & 7.49 \\
34,500 & 18.92 \\
6,310 & 10.30 \\
3,000 & 5.26 \\
4,260 & 9.19 \\
3,780 & 8.48 \\
43,300 & 19.51 \\
4,190 & 9.30 \\
56,900 & 17.99 \\
14,500 & 11.80 \\
23,000 & 19.12 \\
25,700 & 20.68 \\
1,720 & 8.60 \\
36,900 & 17.28 \\
22,000 & 19.23 \\
378 & 2.99 \\
3,850 & 9.09 \\
$5,, 870$ & 7.39 \\
3,000 & 5.20 \\
15,700 & 10.80 \\
1,990 & 8.54 \\
8,270 & 11.80 \\
24,200 & 20.03 \\
3,920 & 4.05 \\
16,800 & 14.23 \\
45,000 & 22.70 \\
11,500 & 14.79 \\
4,800 & 13.10 \\
8,420 & 10.82 \\
30,100 & 19.27 \\
1,400 & 6.04 \\
52,100 & 15.50 \\
496 & 6.30 \\
1,430 & 4.90 \\
39,900 & 15.25 \\
16,400 & 16.48 \\
8,050 & 12.60 \\
21,100 & 11.35 \\
16,500 & 14.47 \\
22,000 & 18.05 \\
& \\
& \\
\hline &
\end{tabular}

8.75
19.83

6.86

6.86
30.67
5.89
16.54

16.54

17.53

18.92

5.26

8.48
9.51

9.30
7.99

11.80

20.68

17.28

2.99

9.09
7.39

5.20
0.80

$(10.80$

4.05

4.23 
Station

Lati- Longi- Drainage

Median flow

Stage Discharge

$\left({ }^{\circ},{ }^{\circ}\right)\left({ }^{\circ},{ }^{\circ}\right)$ (ft) $\left(\mathrm{ft}^{3} / \mathrm{s}\right)$ 100-year flood year flood

Discharge above median
03039200
03040000

03041500

03042000

03042200

03042500

03045000

03045500

03049000

03070500

03071000

03072000

03072840

03072880

03074300

03078000

03078500

03080000

03081000

03082200

03083000

03084000

03084500

03085500

03100000

03101000

03103000

03104500

03106000

03108000

03111150

04213200 South Fork Tenmile Cre

Turt le

Brush Run near Buffalo

Mill Creek at Erie lear Run near Buckstown

ittle Conemaugh River at East Conemaugh

Conemaugh River at Seward

作

wo Lick Creek at Graceton

oyalhanna

oyalhanna Creek at New Alexandria

Kiskiminetas River at Avonmore

Buffalo Creek near Freeport

Big Sandy Creek at Rockville, wV

heat River near Pisgah, WV

Dunkard Creek at Shanopin

Georges Creek at Smithfield

enmile Creek near Clarks

Browns Creek near Nineveh at Jefferson

Redstone Creek at Waltersburg

Crantsvill

Casselman River at Markleton

aurel Hill Creek at Ursina

Youghiogheny River below Confluence

oplar Run near Normalvill

llsville

Green Lick Run at Green Lick Reservoir

Gillespie Run near Sutersvill

ig Sewickley Creek near Ambridge

Sugar Run at Pymatuning Dam

ittle Shenango River at Greenville

Pymatuning Creek near Orangeville

Shenango River at Sharon

Conno Rsing

Raccon

Raccoon Creek near West Springfield
AREA

C--Continued

40037850

$\begin{array}{llll}40 & 17 & 78 & 55 \\ 40 & 21 & 78 & 53\end{array}$

$\begin{array}{llll}40 & 25 & 79 & 02\end{array}$

$\begin{array}{llll}40 & 28 & 79 & 11 \\ 40 & 37 & 79 & 10\end{array}$

$\begin{array}{llll}40 & 34 & 78 & 57\end{array}$

$\begin{array}{lll}4031 & 79 & 10\end{array}$

$\begin{array}{llll}40 & 28 & 79 & 12\end{array}$

$\begin{array}{llll}40 & 18 & 79 & 20 \\ 40 & 24 & 79 & 26\end{array}$

$\begin{array}{lllll}40 & 32 & 79 & 28 & 1,72\end{array}$

$\begin{array}{llll}40 & 23 & 79 & 42\end{array}$

$\begin{array}{lllll}40 & 31 & 79 & 56 & 5.78\end{array}$

$\begin{array}{llllll}39 & 36 & 79 & 47 & 1,354\end{array}$

$\begin{array}{rrrrr}39 & 36 & 79 & 47 & 1,354 \\ 39 & 46 & 79 & 58 & 229\end{array}$

$\begin{array}{llll}39 & 48 & 79 & 48\end{array}$

13.3

$\begin{array}{llll}40 & 00 & 80 & 03\end{array}$

17.5

$39 \begin{array}{llll}55 & 80 & 04\end{array}$

$\begin{array}{llll}39 & 52 & 79 & 42\end{array}$

$\begin{array}{llll}39 & 59 & 79 & 46 \\ 39 & 42 & 79 & 08\end{array}$

$\begin{array}{llll}42 & 79 & 08 & 62.5\end{array}$

$\begin{array}{lllll}39 & 52 & 79 & 14 & 34\end{array}$

$\begin{array}{lllll}39 & 49 & 79 & 19 & 121\end{array}$

$\begin{array}{llllll}39 & 50 & 79 & 22 & 1,029\end{array}$

$\begin{array}{llllll}40 & 01 & 79 & 26 & 1,029 & 9.27\end{array}$

40
406 7930

$4014 \quad 7949$

$\begin{array}{llll}40 & 27 & 79 & 43 \\ 40 & 23 & 79 & 46\end{array}$

$\begin{array}{llll}40 & 23 & 79 & 46 \\ 40 & 24 & 80 & 0\end{array}$

$40 \quad 36 \quad 8010$

$\begin{array}{llll}41 & 31 & 80 & 28\end{array}$

$\begin{array}{llll}41 & 30 & 80 & 28 \\ 41 & 25 & 80 & 23\end{array}$

$4119 \quad 8029$

41148031

$\begin{array}{llll}40 & 49 & 80 & 15\end{array}$

$\begin{array}{lllll}40 & 38 & 80 & 20 & 178\end{array}$

$\begin{array}{lllll}40 & 12 & 80 & 24 & 10.3\end{array}$

$\begin{array}{llll}41 & 57 & 80 & 27 \\ 42 & 06 & 80 & 05\end{array}$

4.04
55.39

257.9

15.6

9.34

169
608

356
178

9.16

9.16

4.44
15.02

11.06
19.48

10.51

4.69

13.54

11.20

16.00

34.20

11.58

4.74
13.50

23.20
12.98

10.42

10.42
7.53

17.48

3.14

12.47

5.80

10.94

16.03

5.95
16.68

2.78
7.23

6.28

13.50

6.80
10.70

6.09

12.63

10.15

17.40

14.36
14.28

10.64

7.67
7.03 
Table 2.--Summary of gaging-station data--Continued

[०, degrees; ', minutes; $\mathrm{mi}^{2}$, square miles; ft, feet; $\mathrm{ft}^{3} / \mathrm{s}$, cubic feet per second]

\begin{tabular}{|c|c|c|c|c|c|c|c|c|c|}
\hline $\begin{array}{l}\text { Station } \\
\text { number }\end{array}$ & Station name & $\begin{array}{l}\text { Lati- } \\
\text { tude } \\
\left({ }^{\circ},{ }^{\circ}\right)\end{array}$ & $\begin{array}{l}\text { Longi- } \\
\text { tude } \\
\left({ }^{\circ},{ }^{\circ}\right)\end{array}$ & $\begin{array}{c}\text { Drainage } \\
\text { area } \\
\left(\mathrm{mi}^{2}\right)\end{array}$ & $\begin{array}{l}\frac{\text { Media }}{\text { Stage }} \\
(f t)\end{array}$ & $\frac{\text { an flow }}{\begin{array}{r}\text { Discharge } \\
\left(\mathrm{ft}^{3} / \mathrm{s}\right)\end{array}}$ & $\begin{array}{l}\frac{100-y e}{\text { Stage }} \\
\text { (ft) }\end{array}$ & 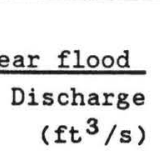 & $\begin{array}{l}\text { Depth of } 100- \\
\text { year flood } \\
\text { above median } \\
\text { stage (ft) }\end{array}$ \\
\hline $\begin{array}{l}01542810 \\
03011800 \\
03012500 \\
03015000 \\
03015080 \\
03015280 \\
03015390 \\
03015500 \\
03017500 \\
03017800 \\
03019000 \\
03020440 \\
030220500 \\
03021350 \\
03021410 \\
03021500 \\
03021700 \\
03022500 \\
03022540 \\
03023500 \\
03024000 \\
03025000 \\
03025200 \\
03026500 \\
03029400 \\
03104760 \\
03106500\end{array}$ & $\begin{array}{l}\text { Waldy Run near Emporium } \\
\text { Kinzua Creek near Guffey } \\
\text { Allegheny River near Kinzua } \\
\text { Conewango Creek at Russell } \\
\text { Akeley Run near Russell } \\
\text { Jackson Run near North Warren } \\
\text { Hare Creek near Corry } \\
\text { Brokenstraw Creek at Youngsville } \\
\text { Tionesta Creek at Lynch } \\
\text { Minister Creek near Truemans } \\
\text { Tionesta Creek at Nebraska } \\
\text { West Branch Caldwell Creek near Grand Valley } \\
\text { Oil Creek at Rouseville } \\
\text { French Creek at Wattsburg } \\
\text { West Branch French Creek near Lowville } \\
\text { French Creek at Carters Corners } \\
\text { Little Conneatee Creek near McKean } \\
\text { French Creek at Saegerstown } \\
\text { Woodcock Creek at Blooming Valley } \\
\text { French Creek at Carlton } \\
\text { French Creek at Utica } \\
\text { Sugar Creek at Sugarcreek } \\
\text { Patchel Run near Franklin } \\
\text { Sevenmile Run near Rasselas } \\
\text { Toms Run at Cooksburg } \\
\text { Harthegig Run near Greenfield } \\
\text { Slippery Rock Creek at Wurtemburg }\end{array}$ & $\begin{array}{ll}\text { AREA } & \text { D } \\
41 & 35 \\
41 & 56 \\
41 & 51 \\
41 & 56 \\
41 & 56 \\
41 & 54 \\
41 & 56 \\
41 & 51 \\
41 & 36 \\
41 & 37 \\
41 & 28 \\
41 & 46 \\
41 & 29 \\
42 & 01 \\
42 & 05 \\
41 & 57 \\
41 & 56 \\
41 & 43 \\
41 & 41 \\
41 & 28 \\
41 & 26 \\
41 & 26 \\
42 & 15 \\
41 & 38 \\
41 & 20 \\
41 & 11 \\
40 & 53\end{array}$ & $\begin{array}{ll}78 & 18 \\
78 & 43 \\
79 & 00 \\
79 & 08 \\
79 & 06 \\
79 & 06 \\
79 & 14 \\
79 & 39 \\
79 & 19 \\
79 & 03 \\
79 & 09 \\
79 & 23 \\
79 & 34 \\
79 & 42 \\
79 & 47 \\
79 & 51 \\
79 & 53 \\
80 & 05 \\
80 & 09 \\
80 & 03 \\
80 & 01 \\
79 & 57 \\
79 & 53 \\
79 & 51 \\
78 & 35 \\
79 & 13 \\
80 & 20 \\
80 & 14\end{array}$ & $\begin{array}{c}5.24 \\
46.4 \\
2,179 \\
816 \\
9.64 \\
12.8 \\
12.3 \\
321 \\
233 \\
10.8 \\
469 \\
4.37 \\
300 \\
92.0 \\
52.3 \\
208 \\
3.60 \\
629 \\
31.1 \\
998 \\
1,028 \\
166 \\
5.69 \\
7.84 \\
12.6 \\
2.26 \\
398\end{array}$ & $\begin{array}{l}3.47 \\
2.45 \\
8.40 \\
3.38 \\
.43 \\
1.98 \\
3.40 \\
2.40 \\
1.60 \\
.65 \\
1.38 \\
5.82 \\
2.45 \\
3.97 \\
3.90 \\
2.93 \\
1.35 \\
3.25 \\
5.96 \\
1.72 \\
3.00 \\
2.82 \\
3.04 \\
2.04 \\
1.38 \\
1.17 \\
1.28\end{array}$ & $\begin{array}{c}2.7 \\
45 \\
2,100 \\
880 \\
9.5 \\
11.8 \\
11.5 \\
280 \\
235 \\
9.5 \\
390 \\
4.0 \\
260 \\
110 \\
60 \\
180 \\
2.2 \\
510 \\
29.4 \\
830 \\
920 \\
135 \\
5.2 \\
7.0 \\
7.9 \\
250.82\end{array}$ & $\begin{array}{r}6.80 \\
9.15 \\
20.20 \\
13.00 \\
4.70 \\
5.55 \\
7.05 \\
13.50 \\
11.35 \\
5.20 \\
10.68 \\
9.40 \\
11.60 \\
13.75 \\
11.10 \\
13.20 \\
4.82 \\
17.75 \\
11.60 \\
15.15 \\
15.35 \\
12.50 \\
5.88 \\
5.73 \\
5.50 \\
4.00 \\
13.30\end{array}$ & $\begin{array}{r}945 \\
6,360 \\
65,500 \\
24,300 \\
1,150 \\
1,110 \\
2,300 \\
17,000 \\
15,600 \\
1,150 \\
20,000 \\
640 \\
19,100 \\
9,240 \\
6,370 \\
20,000 \\
893 \\
26,000 \\
3,080 \\
31,700 \\
32,000 \\
11,400 \\
1,140 \\
3,250 \\
1,140 \\
21,300\end{array}$ & $\begin{array}{r}3.33 \\
6.70 \\
11.80 \\
9.62 \\
4.27 \\
3.57 \\
3.65 \\
11.10 \\
9.75 \\
4.55 \\
9.30 \\
3.58 \\
9.15 \\
9.78 \\
7.20 \\
10.27 \\
3.47 \\
14.50 \\
5.64 \\
13.43 \\
12.35 \\
9.68 \\
2.84 \\
3.69 \\
4.12 \\
2.83 \\
12.02\end{array}$ \\
\hline
\end{tabular}


Table 2.--Summary of gaging-station data--Continued

$\left[{ }^{\circ}\right.$, degrees; ', minutes; $\mathrm{mi}^{2}$, square miles; $\mathrm{ft}$, feet; $\mathrm{ft}^{3} / \mathrm{s}$, cubic feet per second]

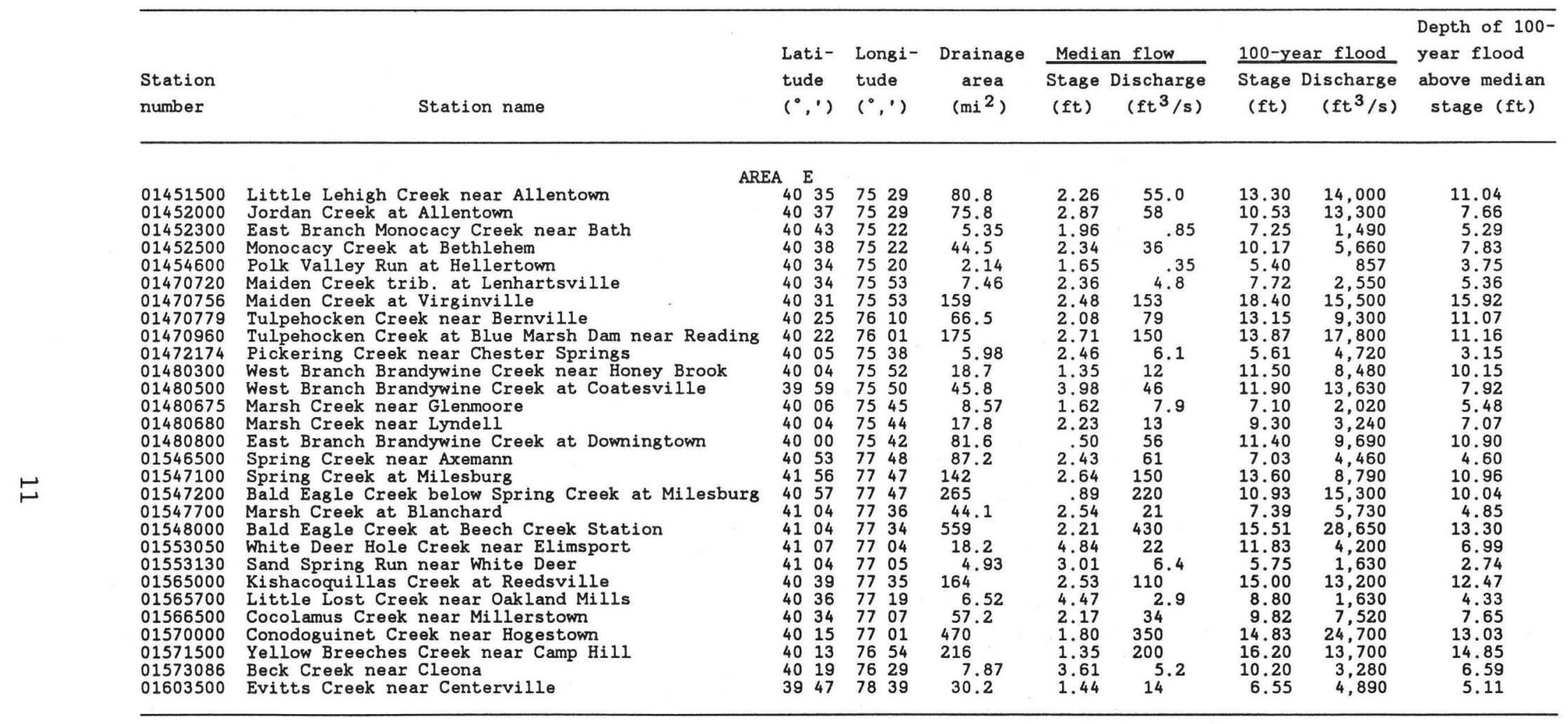

1 Additional information on the locations and records of active gaging stations is given in Water Resources Data for Pennsylvania (U.S. Geological Survey, 1984). 


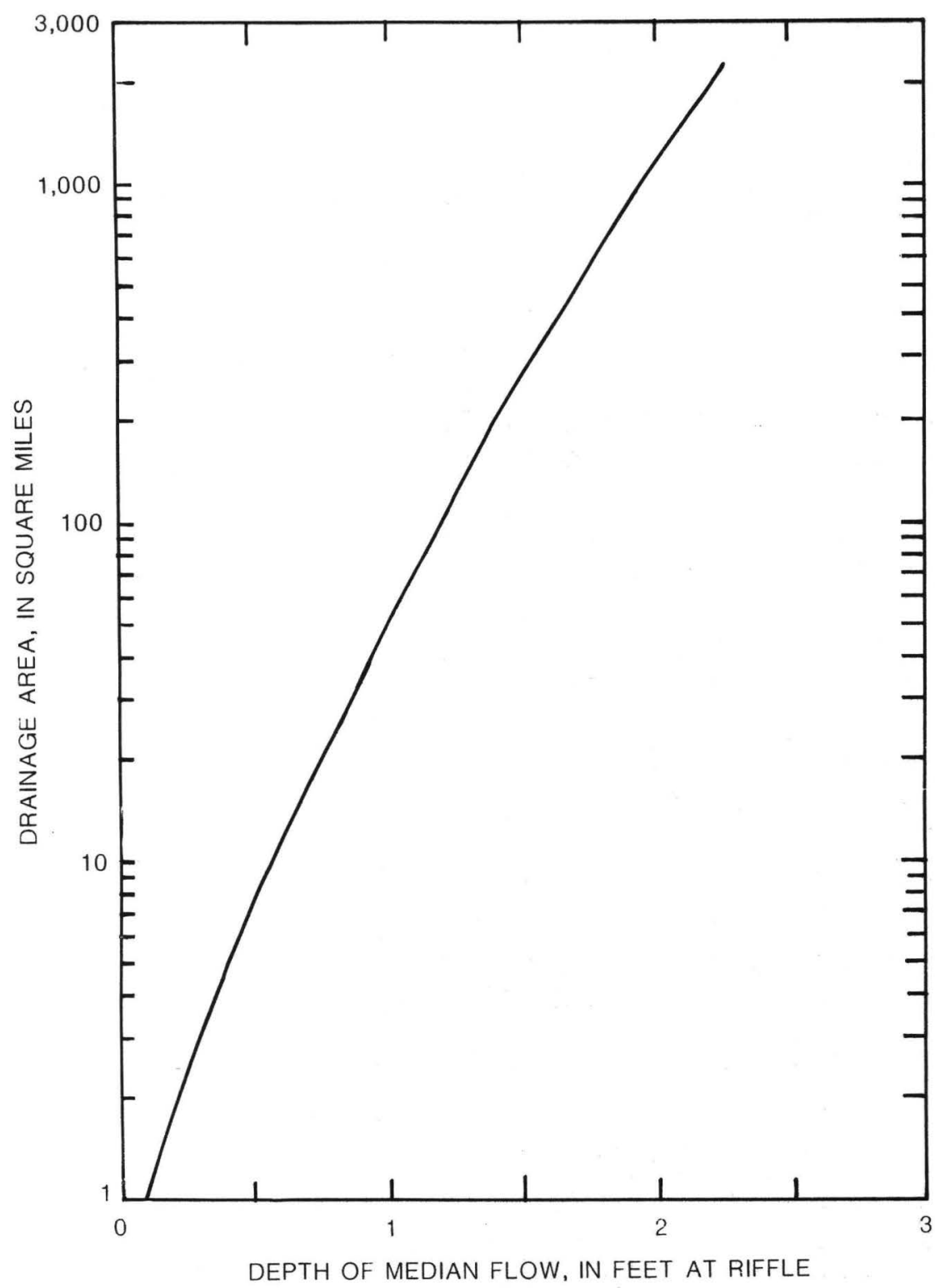

Figure 2.--Relation between drainage area and median depth of flow on riffle.

\section{Application of Results}

Determinations of total flood depth are required if flood-inundation maps are to be prepared or if profiles are to be plotted to show flood depth over the streambed. The procedure for estimating the depth of the 100-year flood above median stage, at a given site on a stream, is as follows:

1. Determine the drainage area, in square miles, of the basin above the site of interest.

2. Determine the hydrologic area for the site from plate 1.

3. Compute the 100-year flood depth $\left(D_{100}\right)$ from the appropriate areal equation (table 1 ) or, alternatively, from the appropriate graph in figure 1 . 
In order to determine the total 100-year depth at the site of interest, it is necessary to add the depth at median stage to the $D_{100}$ depth, as determined by the above procedure. The depth at median stage can be estimated from figure 2 , which is of statewide applicability.

Elevations of the 100-year flood at, or in the vicinity of, the site of interest can be determined by adding the total 100-year depth to the elevation of the streambed at its lowest point on natural riffles. Elevations for such riffle points are best determined by spirit- or electronic-level surveys. High-quality contour maps with contour intervals of 2 feet or less can provide adequate elevation control for flood profiling or mapping of most small, steep-gradient streams.

Flood profiles and maps of lesser accuracy can be developed from 7-1/2 minute U.S. Geological Survey topographic maps, which are available for all of Pennsylvania at contour intervals of 10 or 20 feet. It is commonly assumed that elevations of median stages coincide with the elevations of contour lines where they cross a stream. Thus, depths $\left(D_{100}\right)$ estimated from figure 1 can be added to contour elevations to estimate 100-year-flood elevations at the points of contour crossings. Elevations for flood profiles that are developed in this manner can be no more accurate than the topographic map used. Thirdorder leveling at several localities in Pennsylvania has shown that position errors for these contour lines commonly exceed a quarter of a contour interval, and some may exceed half of a contour interval.

\section{Accuracy and Limitations}

The accuracy of each flood-depth equation (table 1) was measured by comparing the 100-year depth computed through use of the equation with observed depths (table 2) at gaged sites in the delineated ( 1 1. 1) hydrologic areas. Standard errors of estimate for the five equations range from 16 to 28 percent, as shown in table 1 . Two out of three depth $\left(D_{100}\right)$ estimates can be expected to be in error by no more than the standard error of the equation used.

The depth equations should be applied only for drainage areas that fall within the size ranges shown in table 1 . Each applicable range closely matches the range of drainage-area values used in the respective regression. The upper limits of the applicable ranges for areas A and E (325 and $560 \mathrm{mi}^{2}$, respectively) approximate those of the largest unregulated streams in these two areas. Most streams that drain more than 2,200 $\mathrm{mi}^{2}$--the upper limit of the applicable ranges for areas B, C, and D--are regulated.

Depth estimates for sites whose basins lie in more than one of the delineated hydrologic areas (p1. 1) can be proportioned according to the fraction of basin within each area.

The depth equations should not be applied for streams that have been channeled or are highly regulated. The equations should not be used for streams that drain urbanized areas or for those that have more than 15 percent of their drainage controlled by reservoirs, ponds, or swamps. This suggested limit on the percentage of controlled area is based on studies by Aron and 
Kibler (1981, fig. 5-3). Their studies show the reduction in 100-year discharge resulting from 15 percent of controlled area is usually in the range of 5 to 13 percent. The corresponding range in percent of reduction in flood depths will be somewhat less. The relationship for area A incorporates the moderating effects of ponds and swamps on 100-year flood depths in streams that drain the small watersheds that are typical of the Pocono Mountain area.

A constriction or a hydraulic structure, such as a bridge, culvert, or dam, will usually cause a backwater condition on the upstream side of the constriction or structure. The 100-year depth in the reach of backwater will usually exceed that predicted through use of figure 2 and the areal equation of table 1 .

The standard error of the median-depth relation (fig. 2) is $0.25 \mathrm{ft}$. About two-thirds of median-depth estimates made through use of this figure will be in error by less than that amount; however, about 90 percent of measured median depths for drainage areas of less than $10 \mathrm{mi}^{2}$ are within $0.25 \mathrm{ft}$ of the depths indicated by figure 2. For drainage areas greater than $10 \mathrm{mi}^{2}$, about 96 percent of median-depth estimates will be in error by less than two standard errors of estimate, or $0.50 \mathrm{ft}$. Median-depth relations for the five individual hydrologic areas ( 1 1. 1) showed no significant deviation from figure 2, which is based on all available riffle-depth data for the group of gaging stations (table 2). 
Estimates of 100-year flood depth on the riffles in a natural, unregulated Pennsylvania stream can be made by using two relations presented in this report.

Standard errors of estimate for the relations between 100-year depths above median stage and drainage area range from 16 to 28 percent for the five delineated hydrologic areas. An additional error of less than $0.25 \mathrm{ft}$ is typical for estimates of the median-depth component of 100 -year depths on riffles. 


\section{REFERENCES CITED}

Aron, G., and Kibler, D.F., 1981, Procedure PSU-IV for estimating design flood peaks on ungaged Pennsylvania watersheds: The Pennsylvania State Univ. Institute for Research on Land \& Water Resources, Report FHWA/PA 81-013, 211 p.

Flippo, H.N., Jr., 1977, Floods in Pennsylvania: Pennsylvania Department of Environmental Resources, Water Resources Bulletin 13, 59 p.

-.-1982, Evaluation of the streamflow-data program in Pennsylvania:

U.S. Geological Survey Water-Resources Investigations Report 82-21, 56 p.

Hydrology Subcommittee, Interagency Advisory Committee on Water Data, 1982, Guidelines for determining flood flow frequency: U.S. Water Resources Council Bulletin 17B (revised), Washington, D.C., 183 p.

Leopold, L.B., and Maddock, Thomas, Jr., 1953, The hydraulic geometry of stream channels and some physiographic implications: U.S. Geological Survey Professional Paper 252, 57 p.

Riggs, H.C., 1973, Regional analysis of streamflow characteristics: U.S. Geological Survey Techniques of Water-Resources Investigations, Book 4, Chap. B3, 15 p.

U.S. Geological Survey, 1984, Water resources data for Pennsylvania, water year 1984: U.S. Geological Survey Water Data Reports PA-84-1, PA-84-2, PA-84-3. 